\title{
Estudos citogenéticos em formigas neotropicais do gênero Gnamptogenys Roger (Hymenoptera, Formicidae, Ectatomminae)
}

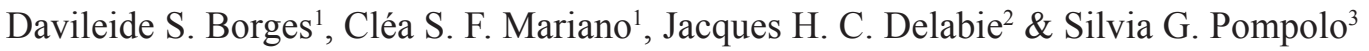

\begin{abstract}
'Departamento de Biologia Animal, Pós Graduação em Entomologia, Universidade Federal de Viçosa. 36570-000 Viçosa-MG, Brasil. Endereço eletrônico: davy@insecta.ufv.br, cmariano@nuxnet.com.br

${ }^{2}$ Unidade de Pesquisa Associada Laboratório de Mirmecologia, Convênio UESC-CEPEC, Centro de Pesquisas do Cacau, CEPLAC. Caixa Postal 7, 45600-000 Itabuna-BA, Brasil. Endereço eletrônico: delabie@cepec.gov.br

${ }^{3}$ Departamento de Biologia Geral. Universidade Federal de Viçosa, 36570-000, Viçosa-MG, Brasil.

Endereço eletrônico: spompolo@mail.ufv.br
\end{abstract}

\begin{abstract}
Cytogenetic studies in Neotropical ants of the genus Gnamptogenys Roger (Hymenoptera, Formicidae, Ectatomminae). Studies on the karyotypes of three Neotropical species of the genus Gnamptogenys (Ectatomminae, Ectatommini) have been carried out: Gnamptogenys striatula Mayr, Gnamptogenys sp. and Gnamptogenys annulata Mayr, collected at Viçosa (Minas Gerais, Brazil) and Ilheus (Bahia, Brazil). The chromosome number of G. striatula was established from individuals taken in colonies from both localities was $2 \mathrm{n}=34$, with the karyotype formula $2 \mathrm{~K}=24 \mathrm{M}+10 \mathrm{~A}$. In Gnamptogenys sp., the chromosomal number was $2 \mathrm{n}=46$ (females) and $\mathrm{n}=23$ (males), and its karyotype formula was $2 \mathrm{~K}=18 \mathrm{M}+28 \mathrm{~A}$. The chromosomal number of $G$. annulata was $2 \mathrm{n}=68$ with the karyotype $2 \mathrm{~K}=6 \mathrm{M}+62 \mathrm{~A}$. This study complements those carried out by our research group on cytogenetics of the poneromorph ants (sensu Bolton) and would contribute to the better understanding of the ant evolution in this group considered primitive.
\end{abstract}

KEYwORDs. Chromosomes; Ectatomminae; Karyotype; Neotropical.

Resumo. Foram caracterizados os cariótipos de três espécies neotropicais do gênero Gnamptogenys Roger: Gnamptogenys striatula Mayr, Gnamptogenys sp. e Gnamptogenys annulata Mayr, coletadas em Viçosa (Minas Gerais) e Ilhéus (Bahia). O número cromossômico de G. striatula nas duas localidades foi $2 \mathrm{n}=34$, com fórmula cariotípica $2 \mathrm{~K}=24 \mathrm{M}+10 \mathrm{~A}$. Em Gnamptogenys sp., o número cromossômico foi de $2 \mathrm{n}=46$ (fêmea) e $\mathrm{n}=23$ (machos), com a fórmula cariotípica $2 \mathrm{~K}=18 \mathrm{M}+28 \mathrm{~A}$. O número cromossômico de $G$. annulata foi $2 \mathrm{n}=68 \mathrm{com}$ a fórmula cariotípica $2 \mathrm{~K}=6 \mathrm{M}+62 \mathrm{~A}$. Esse tipo de estudo complementa outros estudos iniciados por nosso grupo sobre a citogenética das formigas poneromorfas (sensu Bolton) e poderá contribuir no melhor entendimento da evolução das formigas deste grupo considerado primitivo.

Palavras-Chave. Cariótipo; Cromossomos; Ectatomminae; Neotropical.

A recém criada subfamília Ectatomminae (Bolton 2003) é constituída de espécies consideradas primitivas entre os Formicidae (LATTKE 1994, 1995), tanto do ponto de vista morfológico, quanto comportamental. Segundo Bolton (2003), esta subfamília inclui formalmente os gêneros: Ectatomma Fr. Smith, 1858, endêmico à Região Neotropical (LATTKE 1994, 2003), Gnamptogenys Roger, 1863, distribuído nas Regiões Australiana, Neártica, Neotropical e Oriental (BRown 1958; LattKe 1994, 1995, 2002, 2003), Rhytidoponera Mayr, 1862, presente nas Regiões Australiana e Oriental (LatTKe 1994), todos os três colocados na tribo Ectatommini, além de Typhlomyrmex Mayr, 1862, endêmico à Região Neotropical (LATTKe 2003) e único gênero da tribo Typhlomyrmecini. O gênero Gnamptogenys, em particular, conta com 84 espécies descritas na Região Neotropical, as quais mostram grande diversidade de adaptações morfológicas e ecológicas (Brandão \& LattKe 1990; LattKe 1990, 2003; Morgan et al. 2003; PACHECO et al. 2004). As espécies nidificam no solo, em cupinzeiros abandonados ou ativos, madeira podre ou mesmo em epífitas, e seus ninhos têm normalmente menos de 500 operárias (LATTKE 1995). São geralmente monogínicas, mas as colônias de algumas espécies do gênero Gnamptogenys podem abrigar várias fêmeas reprodutoras, como é o caso de Gnamptogenys striatula Mayr, 1884 (GIRAUD et al. 2000).
A citogenética constitui uma ferramenta adicional aos estudos dos mecanismos envolvidos nos processos evolutivos das espécies. Sua aplicação é considerada cada vez mais importante para estudos de filogenia, taxonomia, mecanismos de especiação e da variabilidade genética (GoMES 1995). Os parâmetros utilizados nos estudos citogenéticos são número, morfologia e estruturas (Quantidade de heterocromatina e composição de pares de bases, obtidos por meio de técnicas de bandamentos) dos cromossomos.

Já foram analisados os cariótipos de mais de 500 espécies na família Formicidae, a mais estudada do ponto de vista citogenético entre os Hymenoptera (IMAI et al. 1988; 1994, MARIANO et al. 2003a), e certamente, entre todas as famílias de insetos. Os resultados indicam que as espécies de formigas apresentam amplas variações numéricas nos cariótipos e estruturais nos seus cromossomos. Por exemplo, o número de cromossomos conhecidos varia de $2 \mathrm{n}=2$ em Myrmecia croslandi Taylor,1991 (Myrmeciinae) (Crosland \& Crozier 1986) a 2n=106 em Dinoponera lucida Emery, 1901 (Ponerinae) (MARiano et al. 2004).

São raros os estudos citogenéticos já realizados sobre a subfamília Ectatomminae e, até o momento, nenhuma informação existe sobre qualquer espécie neotropical deste grupo. São conhecidos os números cromossômicos de apenas 
três espécies do complexo Rhytidoponera (metallica) (nomenclatura seguindo as recomendações de OGATA \& TAYLOR 1991): $\mathrm{n}=12,17-22$; Rhytidoponera tasmaniensis (Emery), $\mathrm{n}=23$; Rhytidoponera victoriae (André, 1896), $\mathrm{n}=21$ (Crozier 1969, 1975). Também são conhecidos os números cromossômicos de quatro espécies orientais do gênero Gnamptogenys, que são Gnamptogenys menadensis (Mayr, 1887) $(2 \mathrm{n}=42)$, Gnamptogenys binghamii (Forel, 1900) $(\mathrm{n}=22)$ (ImAi et al. 1983) da Malásia, Gnamptogenys sp.1 (2n=42), Gnamptogenys sp.2 $(2 \mathrm{n}=36)$, ambas do oeste da Malásia e Cingapura (GõNI et al. 1982; IMAI 1983).

O objetivo do presente estudo foi de caracterizar os cariótipos de algumas espécies neotropicais de Gnamptogenys e inferir sobre as possíveis variações numéricas e morfológicas observadas no gênero.

\section{MATERIAL E MÉTODOS}

Coletas de material biológico foram realizadas em Viçosa, MG e Ilhéus, BA. Em Viçosa, as coletas foram realizadas na Mata do Paraíso $\left(20^{\circ} 45^{\prime} \mathrm{S} 45^{\circ} 52^{\prime} \mathrm{W}\right)$, área de Reserva Natural da Universidade Federal de Viçosa, com cerca de 194 hectares e altitude média de $650 \mathrm{~m}$. A vegetação é de Mata Atlântica secundária do subtipo Floresta Subcaducifólia Tropical (Alonso 1977). Em Ilhéus, foram realizadas coletas nos campos experimentais do Centro de Pesquisas do Cacau (CEPEC) da CEPLAC ( $\left.14^{\circ} 15^{\prime} \mathrm{S} 39^{\circ} 13^{\prime} \mathrm{W}\right)$, com altitude média de $40 \mathrm{~m}$, em cacauais incluídos no bioma Mata Atlântica da Região Nordeste.

Foram coletadas 16 colônias de Gnamptogenys, 13 na Mata do Paraíso (março de 2002 e abril de 2003) e as três restantes na segunda localidade (julho e agosto de 2002). As espécies identificadas foram as seguintes: G. striatula (12 colônias, sendo 10 em Viçosa e 2 em Ilhéus), Gnamptogenys sp. (2 colônias em Viçosa), Gnamptogenys annulata (Mayr, 1887) (1 colônia em Ilhéus). Espécimens destas espécies foram depositados na Coleção do Laboratório de Mirmecologia do CEPEC, Ilhéus, Bahia.

As colônias foram levadas ao Laboratório de Citogenética de Insetos da Universidade Federal de Viçosa (UFV), abrigadas em ninhos artificiais de gesso com $20 \mathrm{~cm}$ de diâmetro, onde foram mantidas em câmara de incubação (B.O.D. 347 CD) a $26^{\circ} \mathrm{C}$, com a finalidade de obter prépupas logo após a rejeição do meconium (fase "pós-defecante"). As colônias foram alimentadas com larvas de Tenebrio molitor Linneaus, 1758 (Coleoptera, Tenebrionidae) e mel.

A análise citogenética foi feita a partir de gânglios cerebrais destas prépupas, seguindo a metodologia de IMAi et al. (1988), cujo resultado consegue informar a distribuição de heterocromatina, não sendo necessária a utilização de técnica de banda-C (ImAi et al. 1988). Em média, foram analisadas 10 lâminas por colônia, que foram coradas com Giemsa diluído em tampão Sörensen na proporção de 1:30 por 20 minutos. As metáfases foram observadas e fotografadas num microscópio Olympus BX-60, com objetiva de imersão de 100x e filtro verde, acoplado a um sistema de fotografia.

Os caracteres observados foram o número, a morfologia dos cromossomos e a distribuição da heterocromatina. A representação cariotípica foi feita de acordo com a nomenclatura de IMAI (1991). Para a montagem dos cariótipos, os cromossomos foram classificados em dois grupos: grupo M, formado de cromossomos metacêntricos (M), cuja heterocromatina se localizava nas regiões pericentroméricas; e grupo A, de cromossomos acrocêntricos (A), cuja heterocromatina se localizava em toda a extensão do braço curto dos cromossomos. Neste segundo grupo, estão incluídos os cromossomos pseudo-acrocêntricos $\left(\mathrm{A}^{\mathrm{M}}\right)$ [seguindo a nomenclatura de IMAI (1991)] com um braço longo heterocromático.

\section{RESULTADOS}

O número cromossômico encontrado para $G$. striatula foi de $2 n=34$, tanto nas colônias coletadas em Viçosa, quanto naquelas coletadas em Ilhéus. A técnica de coloração convencional com Giemsa não permitiu evidenciar diferenças na constituição dos cariótipos entre colônias coletadas nas duas localidades. O cariótipo desta espécie é composto por 12 pares de cromossomos metacêntricos $(\mathrm{M})$ e cinco pares de cromossomos acrocêntricos (A), com fórmula cariotípica $2 \mathrm{~K}=24 \mathrm{M}+10 \mathrm{~A}$ (Fig. 1a).

Em Gnamptogenys sp., o número cromossômico encontrado em todas as observações foi de $2 n=46$ para fêmea (Fig. 1b) e $n=23$ para machos, sendo o cariótipo composto de

Tabela I. Informações citogenéticas disponíveis sobre o gênero Gnamptogenys. 2n(n): número de cromossomos diplóide [2n], haplóide [n]; $2 \mathrm{~K}(\mathrm{~K})$ : fórmula cariotípica diplóide $[2 \mathrm{~K}]$, haplóide $[\mathrm{K}]$.

\begin{tabular}{|c|c|c|c|c|}
\hline Taxon & Localidade, país & $2 n(n)$ & $2 \mathrm{~K}(\mathrm{~K})$ & Referência \\
\hline G. annulata & Ilhéus, BA, Brasil & 68 & $2 \mathrm{~K}=6 \mathrm{M}+62 \mathrm{~A}$ & o presente estudo \\
\hline G. binghami & Malásia & $(22)$ & não definido & IMAI et al. 1983 \\
\hline G. menadensis & Malásia & 42 & não definido & IMAI et al. 1983 \\
\hline G. striatula & Ilhéus, BA, Brasil & 34 & $2 \mathrm{~K}=24 \mathrm{M}+10 \mathrm{~A}$ & o presente estudo \\
\hline G. striatula & Viçosa, MG, Brasil & 34 & $2 \mathrm{~K}=24 \mathrm{M}+10 \mathrm{~A}$ & o presente estudo \\
\hline Gnamptogenys sp. & Viçosa, MG, Brasil & $46(23)$ & $2 \mathrm{~K}=18 \mathrm{M}+28 \mathrm{~A}(\mathrm{~K}=9 \mathrm{M}+14 \mathrm{~A})$ & o presente estudo \\
\hline Gnamptogenys sp.1 & Malásia, Cingapura & 42 & não definido & GõnI et al. 1982, IMAI 1983 \\
\hline Gnamptogenys sp.2 & Malásia, Cingapura & 36 & não definido & GÕNI et al. 1982, IMAI 1983 \\
\hline
\end{tabular}


A)

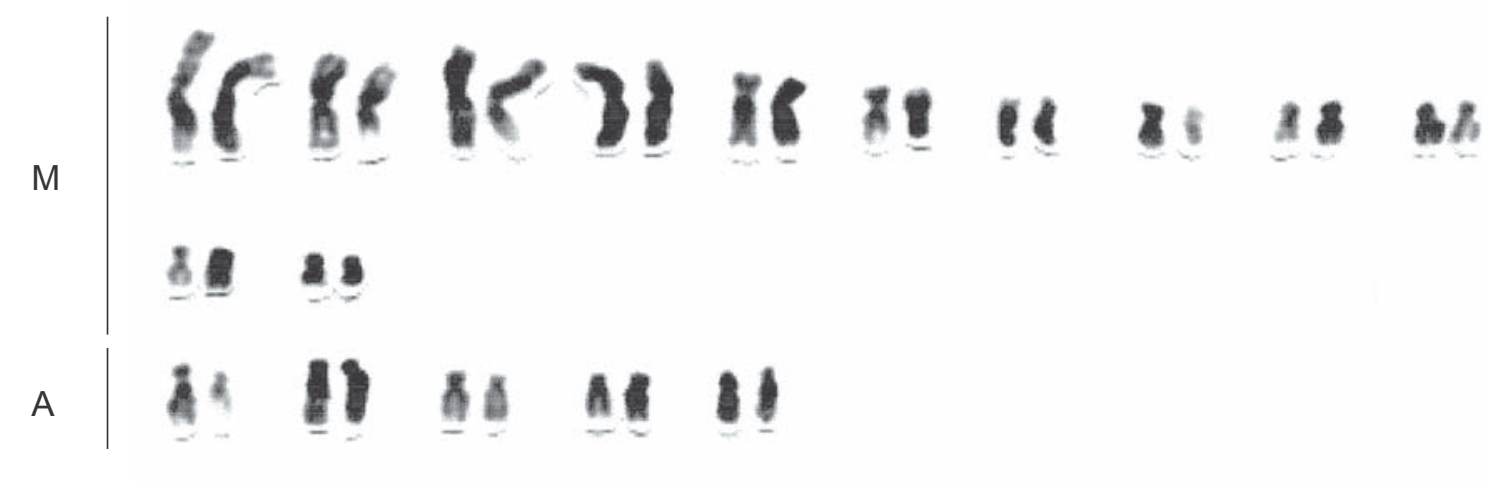

B)

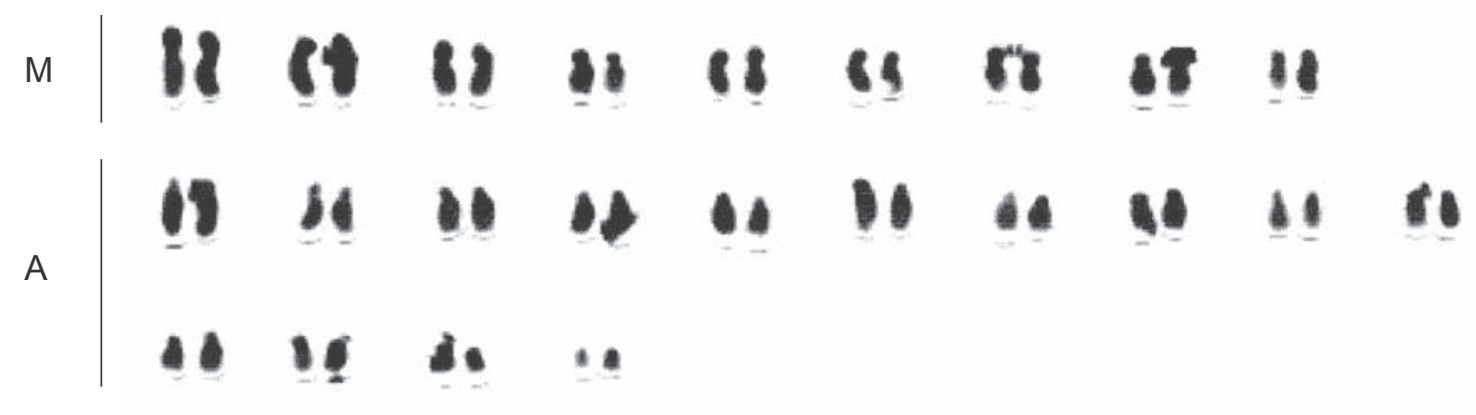

C)
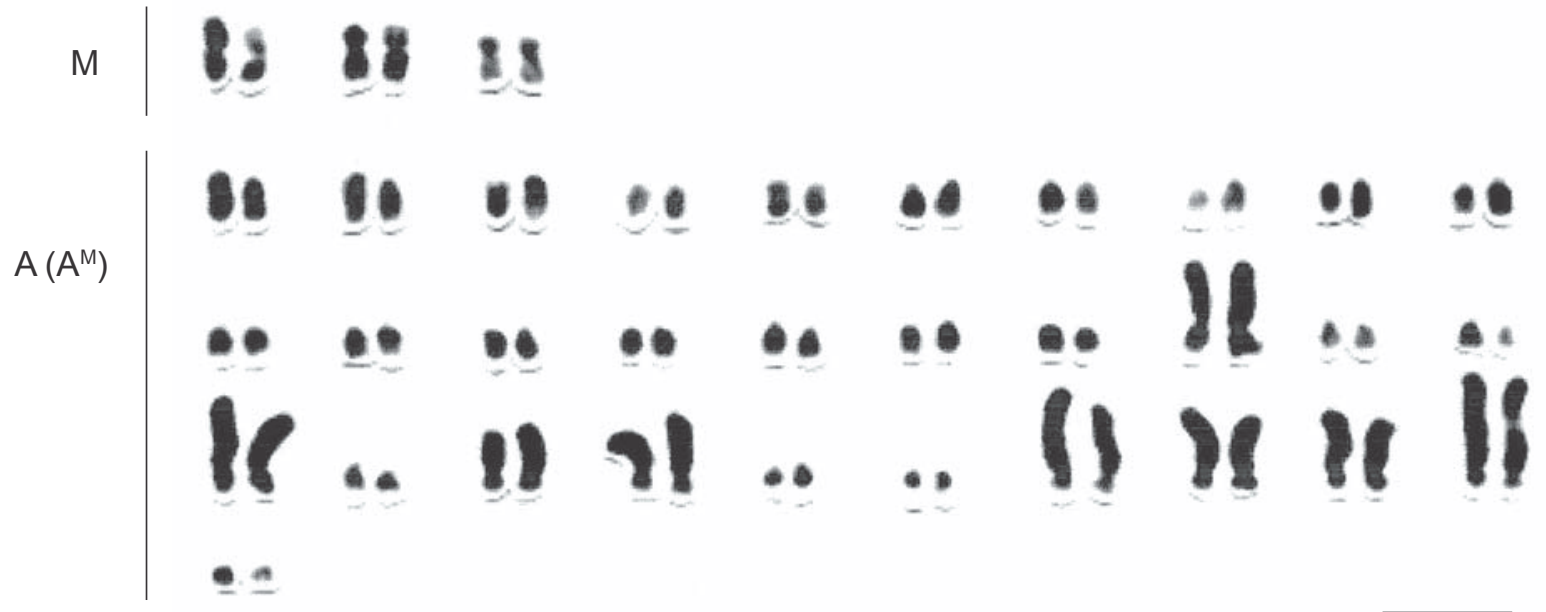

Fig. 1 - Cariótipos diplóides de espécies neotropicais do gênero Gnamptogenys. A) Gnamptogenys striatula, 2n=34; B) Gnamptogenys sp., $2 \mathrm{n}=46$; C) Gnamptogenys annulata, $2 \mathrm{n}=68$. Barra $=5 \mu \mathrm{m}$.

oito pares de cromossomos metacêntricos (M) e 15 pares de cromossomos acrocêntricos (A), $2 \mathrm{~K}=16 \mathrm{M}+30 \mathrm{~A}$ para as fêmeas e $\mathrm{K}=8 \mathrm{M}+15 \mathrm{~A}$ para os machos.

O número cromossômico encontrado na análise de $G$. annulata foi $2 \mathrm{n}=68$ (Fig. 1c), com três pares de cromossomos metacêntricos (M) e 31 pares de cromossomos acrocêntricos (A), com fórmula cariotípica de $2 \mathrm{~K}=6 \mathrm{M}+62 \mathrm{~A}$ nas fêmeas. Foi observada a ocorrência de braços nitidamente heterocromáticos em oito pares de cromossomos A, denominados $\mathrm{A}^{\mathrm{M}}$.

\section{DISCUSSÃO}

A Tabela I apresenta, de forma resumida, as informações disponíveis sobre os Gnamptogenys já estudados do ponto de vista citogenético. Entretanto, não foi possível fazer uma análise completa, comparando nossas próprias observações com essas espécies, pois não foi publicado nenhum detalhe sobre sua estrutura cromossômica.

No caso do táxon G. striatula $(2 \mathrm{n}=34)$, não foram encontradas diferenças evidentes entre cariótipos de 
indivíduos oriundos das duas localidades de coleta (Viçosa e Ilhéus, distantes de cerca de $1.000 \mathrm{~km}$ ), demonstrando grande estabilidade cariotípica neste táxon ou sugerindo ainda que esta espécie se expandiu recentemente. Ambas hipóteses não se excluem mutuamente, mas a segunda prevalece uma vez que este taxón é freqüentemente encontrado em meio urbano no Estado da Bahia, deixando supor uma origem similar à de outras espécies que vivem hoje em dia no mesmo ambiente.

Com 84 espécies descritas na Região Neotropical (LATTKE 1994, 1995, 2003), Gnamptogenys ainda é um gênero pouco estudado sob o ponto de vista citogenético. Ao contrário, o gênero Camponotus é considerado o maior em número de espécies com o cariótipo estudado (pelo menos superficialmente, com o número de cromossomos determinado) para toda a família Formicidae (MARIANO et al. 2003b)

Não foram encontrados um cromossomo, ou um grupo de cromossomos, que poderia ser identificado como típico do gênero Gnamptogenys, e que ocorreria, por exemplo, em todas as espécies. Isso leva à questão da necessidade de se fazerem novas abordagens no estudo da citogenética das formigas, tais como procurar adaptar as diversas técnicas de bandamentos cromossômicos existentes (SUMNER 1990) visando à identificação de um possível marcador cromossômico típico deste gênero. Esse tipo de informação, junto aos resultados de diversos estudos iniciados por nosso grupo sobre a citogenética das formigas poneromorfas sensu Bolton (2003) (MARiano et al. 2003a, 2004), poderá contribuir no melhor entendimento da evolução das formigas deste grupo.

Agradecimentos. À FAPEMIG, pela concessão de bolsa a D.S.Borges e ao CNPq e CAPES por diferentes modalidades de auxílios financeiros.

\section{REFERÊNCIAS}

Alonso, M. T. A. 1977. Vegetação. In: C. Goldenberg (ed.). Geografia do Brasil: região sudeste. Rio de Janeiro, IBGE, vol. 3: 91118.

Bolton, B. 2003. Synopsis and classification of Formicidae. Florida, The American Entomological Institute, 370 p.

BRown, W. L. 1958. Contributions toward a reclassification of the Formicidae. II. Tribe Ectatommini. Bulletin of the Museum of Comparative Zoology 118: 175-362.

Brandão, C. R. F. \& J. E. LattKe. 1990. Description of a new Ecuadorean Gnamptogenys species (Hymenoptera: Formicidae), with a discussion on the status of the Alfaria group. Journal of the New York Entomological Society 98(4): 489-494.

Crosland, M. W. J. \& R. H. Crozier. 1986. Myrmecia pilosula, an ant with only one pair of chromosome. Science 231: 1278.

Crozier, R. H. 1969. Chromosome number polymorphism in an
Australian ponerinae ant. Canadian Journal of Genetics and Cytology 2: 333-339.

Crozier, R. H. 1975. Hymenoptera. In: B. John (ed.). Animal Cytogenetics. Berlin, Gebrudeer Borneträeger, (Insecta, 7) 3: 95p.

Giraud, T.; R. Blatrix; C. Poteaux; M. Solignac \& P. Jaisson. 2000. Population structure and mating biology of the polygynous ponerine ant Gnamptogenys striatula in Brazil. Molecular Ecology 9: 18351841.

Gomes, L. F. 1995. Estudos citogenéticos em vespas do gênero Trypoxylon (Hymenoptera, Sphecidae, Larrinae, Trypxylonini). Dissertação de Mestrado. Viçosa, Universidade Federal de Viçosa, 80 $\mathrm{p}$.

GoÑi, B.; H. T. Imai; M. Kubota; M. Kondo; H. Yong \& Y. Р. Тно. 1982. Chromosome observations on tropical ants in western Malaysia and Singapore. Annual Republic of National Institute Genetics of Japan 32: 71-73.

ImAI, H. T. 1991. Mutability of constitutive heterochromatin (C-bands) during eukaryotic evolution and their cytological meaning. Japanese Journal of Genetics 66: 635-661.

Imai, H. T.; W. L. Brown-J. R.; M. Kubota; H. Yong \& Y. P. Tho. 1983. Chromosome observations on tropical ants from western Malaysia II. Annual Republic of National Institute Genetics of Japan 34: $66-69$

Imai, H. T., M. W. J. Crosland; R. H. Crozier; \& R. W. Taylor. 1988. Modes of spontaneous chromosomal mutation and karyotype evolution in ants with reference to the minimum hypothesis. Japanese Journal of Genetics 63: 159-185.

Imai, H. T.; R. H. Crozier \& R. W. Taylor. 1994. Experimental bases for the mininum interaction theory. I Chromosome evolution in ants of the Myrmecia pilosula species complex (Hymenoptera: Formicidae: Myrmeciinae). Japanese Journal of Genetics 69: 137-182.

LatTKE, J. E. 1990. Revision del genero Gnamptogenys Mayr en Venezuela (Hymenoptera: Formicidae). Acta Terramaris 2: 46 p.

LattKe, J. E. 1994. Phylogenetic relationships and classification of ectatommine ants (Hymenoptera: Formicidae). Entomologica Scandinavica 25: 105-119.

LatTKE, J. E. 1995. Revision of the ant genus Gnamptogenys in the New World (Hymenoptera: Formicidae). Journal of Hymenoptera Research 4: 137-193.

Lattke, J. E. 2003. Subfamilia Ponerinae, p. 261-276 In: F. Fernández (ed.) Introducción a las hormigas de la región Neotropical. Bogotá, Instituto de Investigación de Recursos Biológicos Alexander von Humboldt, 398 p.

Mariano, C. S. F.; S. G. Pompolo \& J. H. C. Delabie. 2003a. Citogenética em Formicidae. Anais do XVI Simpósio de Mirmecologia 16: 81-85.

Mariano, C. S. F.; J. H. C. Delabie; L. A. O. Campos \& S. G. Pompolo. 2003 b. Trends in karyotype evolution in the ant genus Camponotus (Hymenoptera: Formicidae). Sociobiology, 42(3): 831-839.

Mariano, C. S. F.; J. H. C. Delabie; L. S. Ramos; S. Lacau \& S. G. Pompolo. 2004. Dinoponera lucida Emery (Formicidae: Ponerinae): largest number of chromosomes known in Hymenoptera. Naturwissenschaften 91: 182-185.

Morgan, C.; W. Mackay \& J. Pacheco. 2003. A new Gnamptogenys of the striatula group from Bolivia (Hymenoptera: Formicidae). Sociobiology 42: 553-558.

Ogata, K. \& R. W. TAYlor. 1991. Ants of the genus Myrmecia Fabricius: a premilinary review and key to named species (Hymenoptera: Formicidae: Myrmiciinae). Journal of Natural History 25: 16231673.

Pacheco, J.; W. Mackay \& C. Morgan. 2004. A new species of Gnamptogenys Roger of the sulcata group (Hymenoptera: Formicidae) from Bolivia. Proceedings of the Entomological Society of Washington 106: 434-437.

Sumner, A. T. 1990. Chromosome banding. $1^{\text {st }}$ ed. London Unwin Hyman Ltd., $434 \mathrm{p}$.

Recebido em 08.III.2004; aceito em 30.VIII.2004 